\title{
Research on Indoor Map Production for Fire Rescue
}

\author{
Ming Wang ${ }^{1,2}$, a ling Peng ${ }^{1, b}$, Yuhuan Ren ${ }^{1}$ \\ $1^{1}$ Institute of Remote Sensing and Digital Earth, CAS, Beijing 100094, China \\ ${ }^{2}$ University of Chinese Academy of Sciences, Beijing 100049, China \\ awangming12@mails.ucas.ac.cn, bpengling@radi.ac.cn
}

\begin{abstract}
Keywords: Fire Rescue; Indoor Map.
Abstract. Indoor map has become a hotspot of electronic map research. However, the usual indoor map production methods couldn't provide useful information for fire rescue. In this paper, by the way of investigating requirements of fire fighting and rescue, analyzing the characteristic of indoor map for fire rescue, and using ARCGIS software in map production, we try to explore a method to product standardized indoor map for fire rescue. It contains preparation, map registration, quality testing, map digitization and map compilation. This method has been used in Tianjin indoor map production for fire rescue.
\end{abstract}

\section{Introduction}

With the development of positioning technology and mobile internet technology, the topic for spatial applications has been changed from the outdoor to indoor. The indoor map has gradually become a hot spot of electronic map research. Google has issued the first indoor positioning application for Android which is based on Google map, contains indoor map of some large public facilities in Japan and America. Scholars also have some academic research on the indoor positioning and navigation $[1,2]$. We base on their research, especially in the field of fire protection [3]. Motivated by indoor map development, the major electronic map manufacturers in china, such as Amap and Baidu has accessed to markets of indoor map.

However, due to the research lack of indoor map in theories and methods [4], the existing indoor map was made by the approach of traditional outdoor map production. It often ignores the data mining and the utilization of the geographic element attribute, and it can't provide clear information to the user to distinguish evaluation. By use of complex indoor architectural structure, high quantity geographic information data, and domain knowledge for users' requirements, the indoor map application service has been change from navigation to data mining. The users pay no attention to how to show the geographic graphics but to use the attribute information of the indoor map. The map should be provided more details, professional information to the users, and the indoor map production need an industry standard. For the practical application in fire fighting and rescue, it needs a professional produce controls and useful information in the indoor map. This article is based on the fire fighting and rescue needs, combined with the production tools of ERSI platform, and it explores a fire indoor map method for fire fighting and rescue application.

According to the need of fire rescue application, the particularity of fire indoor map production will be described in the following three aspects:

1. It contains special symbolic expression of fire information.

These elements are identified in dot icons, such as fire fighting facilities, alarm facilities, smoke control facilities, evacuation facilities, and risk sources. The elements which can't be expressed by dot could be showed in the linear facilities layers, such as fire shutter etc...

2. The map can express the road of the floor by network icons, and it can be used in evacuation and rescue application analysis.

3. It contains the descriptions for wall, door and material in the linear facilities layer. Height and direction information can be detailed recorded, such as the wall can be distinguished by load bearing walls and non load ones, the gate can be distinguished by material, type, and the door direction. It also provides those attribute information. 


\section{Design of fire indoor map}

For the special requirement of the fire protection application, fire indoor map data mainly contains following 5 layers.

1. FIRE POI (Point of Interest)

This layer contains different types of fire POI symbols.

For example, fire fighting facilities, alarm facilities, smoke control facilities, evacuation facilities. 2. LINE

This layer describes symbols of line elements.

For example, walls, glass partition, railings, window, door, fire shutter.

3. DOOR

This layer contains the fire extinguishing, door as point symbols for rescue application.

4. CHANNEL

The linear corridor network symbols for rescue application

5. ROOM

The layer contains polygon elements like room, corridor, with their attribute information

\section{The process of fire indoor map}

\section{Data preparation}

The raw data of fire indoor map comes from building construction plans in the construction drawings and construction blueprint.

The building plans should be converted into Shape-File vector format. Construction blueprint need to be scanned and to be converted into grid scanning map.

2. Map registration

By map registration, the vector or raster format indoor map will obtain the accurate information of geographical coordinates.

3. Map digitizing

After the registration, map elements should be drawn, measured and recorded. In the topology check, we should find out the elements mistakes and ensure that the map data can be correctly performed in data analysis and applications.

4. Quality testing

After the completion of map digitization, we need check on the quality of the indoor map data.

5. Map compilation

The map compilation contains map graphic symbols, note symbol sets, the legend, the compass and other graphic elements configuration.

\section{Fire indoor map making}

\section{Geographic information system platform selection}

In this paper, we choose the commercial software ARCGIS Desktop to map production. It is convenient for data to convert, edit, display and check, and to use personal geo-database format to ensure the accuracy of projection coordinate and topology characteristic.

\section{Preparation and processing of the raw data}

1. Obtaining the following raw data

It contains the building construction plan drawing in CAD format, the construction blueprint, the building base map data, and the registration layer.

2. The data preprocessing

When the raw data is the construction of CAD file, we use the Arc-Tool-Box tool, to make the building plan export to Shape-File format.

When the data is the paper construction blueprint, we need to use raster scanning tool. 


\section{The map registration operation}

With enough accurate registration points, we use the ARCGIS spatial adjustment tool to complete the registration operation.

\section{The map digitization processing}

1. To create the map layer

We use Arc-Catalog to create a new layer. The projection of the layer is set by the projection information consistent with the registration layer. Each layer is named after the rules by floor names: Such as Building names, floor name, layer content identifier, and the "Marking" content in table following. For example, the 2th layer POI layer of A Building is named as A2poi.shp. Table 1 shows the rules of the layers' name.

Table 1. The rules of the layers

\begin{tabular}{|c|c|c|}
\hline Number & Name of layers & Marking \\
\hline 1 & POI & poi \\
\hline 2 & channel & line \\
\hline 3 & room & polygon \\
\hline
\end{tabular}

2. To set the layers' attribute information

We use the bar in Arc-Catalog to add the required attribute field. Table 2 shows a part of attribute field of ROOM layer.

Table 2. Attribute field of ROOM layer

\begin{tabular}{|l|l|l|l|l|l|l|}
\hline Number & Key & Description & Type & Length & The range & The default value \\
\hline 1 & Room-number & Room numbers & text & 50 & $\ldots$ & NULL \\
\hline 2 & Room-type & Type of rooms & text & 50 & $\ldots$ & NULL \\
\hline 3 & Com-name & Company name & text & 50 & $\ldots$ & NULL \\
\hline 4 & description & description & text & 50 & $/$ & NULL \\
\hline$\ldots$ & $\ldots$ & $\ldots$ & $\ldots$ & $\ldots$ & $\ldots$ & $\ldots$ \\
\hline
\end{tabular}

3. To create and edit personal geo-database file

We use the Personal Geo-database which is named by the building name to set the projection and topology checking.

The topology checking is aimed to find out the map drawing errors. The checking rules includes such as:

Must Not Overlap (in the same feature classes, one line and another line cannot overlap each other)

Must Not Intersect (one line and another line can’t intersect the same feature classes)

Must Not Overlap With (two line elements in one class can’t overlap each other)

Must Not Self Overlap (can’t overlap itself)

Must Not Self Intersect (can't intersect itself)

Must Not Intersect or Touch Interior (lines should not cross itself, and no endpoint can be contacted)...

\section{Drawing elements}

We should open Arc-Map and create a new personal geo-database file. To make sure the layer editing status is editing, we need to select the task to create new elements, set the target layer to the editing layer, open the automatic capturing and tracking, and drawing the layer. Figure 1 and Figure 2 show CAD file and the indoor map. 


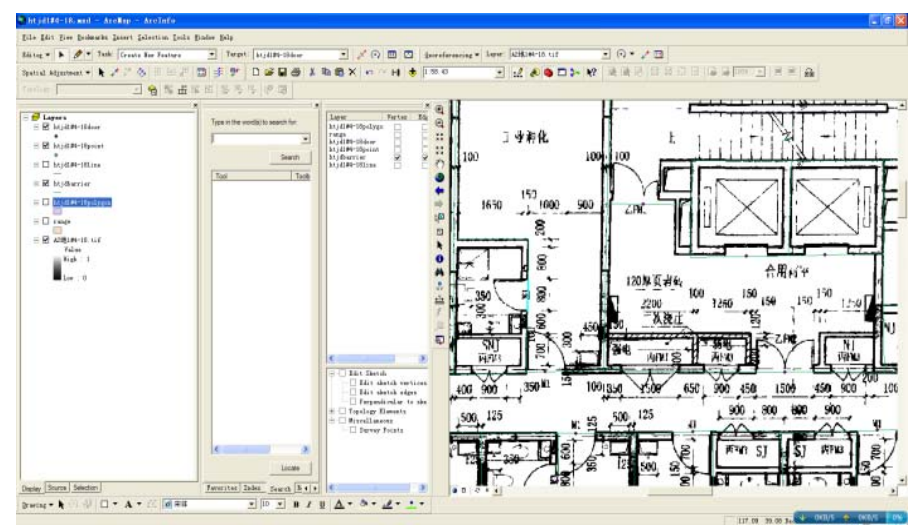

Fig. 1. The CAD file before drawing

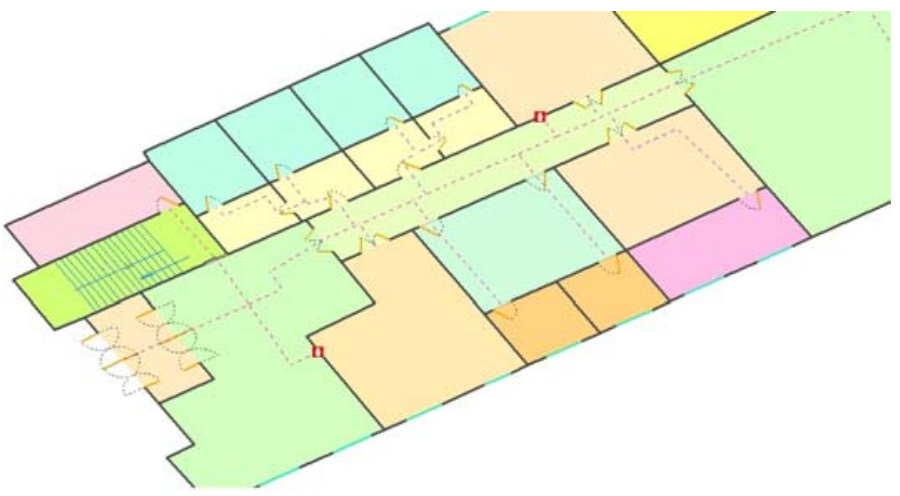

Fig. 2. The fire indoor map after drawing

\section{The quality testing and map compilation}

After the drawing we need test the quality of each layer, including the topology accuracy of graph feature and the correction of attribute information. Map topological features of fire indoor map have influence on path analysis. It is crucial for the actual effect of indoor map application. To check and correct those errors, we need to do a lot of work in outside-work to ensure the data accuracy. By the way of the user declaration platform, the maintenance costs of the map have dropped directly.

The map compilation operation should refer to the specification standard of fire indoor map which contains map graphic symbols, note symbol sets, the legend, the compass and other graphic elements configuration. We also refer to other electronic map drawing specifications to complete the fire indoor map production process.

\section{Conclusion}

The fire fighting and rescue applications make great demands on availability and reliability of the indoor map. Fire fighters ask the fire indoor map for to show the facilities and building structure. With the project of fire indoor map in Tianjin, we collected requirements from fire fighters to produce fire indoor map, learned from traditional map production method and formed fire indoor map production method. It contains data preparation, map registration, digitization processing, quality testing, map digitization and map compilation.

\section{Acknowledgements}

This work was financially supported by the National High Tech Research and development program (2012AA12A401). 


\section{References}

[1] Fuqiang Wang, Zhiwei Xue, Xiaofei Qi, Review of the research on indoor map. Dikuang-cehui, 2012, 28(2): p. 1-3. (in chinese)

[2] Jiayan Hu, Xiuquan Chen, Yinchun Tao, The research on indoor and outdoor location based on LBS. Computer Engineering.C, 2010(8): p. 254. (in chinese)

[3] Xiaoli Ren. The research on indoor navigation based on UWB/GIS technology. 2010, East China Normal University: p. 80. (in chinese)

[4] Xiaofei Qi, Xiushu Cui. The analysis of current indoor map design.Surveying and spatial information 2013, 36(2): p. 10-14. (in chinese) 